\title{
Prevalence of Integrons and Antibiotic Resistance Pattern in Acinetobacter baumannii Isolated from Clinical Samples of Iranian Patients: A Systematic Review and Meta-analysis
}

\section{Mehran Ghazalibina ${ }^{1}$, Hamed Mortazavi ${ }^{2}$, Mahtab Babadi ${ }^{3,4}$, Mohammadreza Rahimi $^{3,4}$, Azad Khaledi ${ }^{3,4}$, Manouchehr Teymouri ${ }^{5}$, Ehsan Saburi ${ }^{6}$}

\section{OPEN ACCESS}

Citation: Mehran Ghazalibina, Mahtab Babadi, Mohammadreza Rahimi, Azad Khaledi, Ehsan Saburi. Prevalence of Integrons and Antibiotic Resistance Pattern in Acinetobacter baumannii Isolated from Clinical Samples of Iranian Patients: A Systematic Review and Metaanalysis. Ethiop $J$ Health Sci.2019;29(5):639.doi:http:// dx.doi.org/ 10.4314/ejhs.v29i5.15

Received: June 9, 2019

Accepted: July 1, 2019

Published: September 1, 2019

Copyright: (C) 2019 Mehran G., et al.

This is an open access article distributed under the terms of the Creative Commons Attribution License, which permits unrestricted use, distribution, and reproduction in any medium, provided the original author and source are credited.

Funding: Nil

Competing Interests: The authors declare that this manuscript was approved by all authors in its form and that no competing interest exists.

Affiliation and Correspondence:

${ }^{1}$ Department of Microbiology, School of Public Health, Tehran University of Medical Sciences, Tehran, Iran

${ }^{2}$ Geriatric Care Research Center, Department of Geriatric Nursing, School of Nursing and Midwifery, North Khorasan University of Medical Sciences, Bojnurd, Iran

${ }^{3}$ Infectious Diseases Research Center, Faculty of Medicine, Kashan University of Medical Sciences, Kashan, Iran

${ }^{4}$ Department of Microbiology and Immunology, Faculty of Medicine, Kashan University of Medical Sciences, Kashan, Iran

${ }^{5}$ Natural Products and Medicinal Research Center, North Khorasan University of Medical Sciences, Bojnurd, Iran

${ }^{6}$ Immunogenetic and Cell Culture Department, Immunology Research

Center, School of Medicine, Mashhad
University of Medical Sciences, Mashhad, Iran

*Email: azadkh99@gmail.com

\section{ABSTRACT}

BACKGROUND: Acinetobacter baumannii is an important opportunistic nosocomial pathogen. Class 1 integrons in A. baumannii plays a significant role in antibiotic resistance. Therefore, this study aimed to investigate the prevalence of integrons and antibiotic resistance pattern in A. baumannii isolated from clinical samples of Iranian patients.

METHODS: The Medical Subject Headings (MeSH) and the keywords with the help of Boolean operators ("AND” or "OR") were used alone or in combination to conduct the search. The searching process was conducted in the Web of Science, PubMed, Cochrane Library, Scopus, and Google Scholar databases and, also Iranian databases. The search was restricted to relevant English and Persian cross-sectional publications reporting the prevalence of Int1 in A. baumannii isolated from clinical samples from 1 January 2000 to 31 December 2018. The data were analyzed using Comprehensive Meta-Analysis software. Regarding the heterogeneity of studies, the random effects model was used. Cochrane $Q$ and $I^{2}$ tests was used to evaluate statistical heterogeneity between the studies.

RESULTS: Fifteen studies were included in the analysis. The combined prevalence of class 1 integrons in A. baumannii was 55.2\% (95\% CI: 44.8-65.1). The pooled prevalence of MDR A. baumannii isolates was $68.1 \%$. The highest resistance belonged to Aztreonam, followed by Ciprofloxacin, and Ceftazidime with a resistance rate of $97.6 \%, 92.8 \%$, and $91.6 \%$, respectively. Tobramycin was reported as an effective antibiotic.

CONCLUSIONS: The present study reported an alarmingly high prevalence of class 1 Integrons, and MDR isolates of $A$. baumannii recovered from clinical samples that should be considered.

KEYWORDS: Prevalence, Integrons, Acinetobacter baumannii, Pathogen 


\section{INTRODUCTION}

Acinetobacter baumannii is an important aerobic Gram-negative opportunistic nosocomial pathogen with broad dissemination in the environment (1). A range of threating nosocomial infections including septicemia, respiratory tract, urinary tract infection, and pneumonia, burn wound and soft tissue are caused by $A$. baumannii especially in the ICU and burn units (2). In the $21^{\text {st }}$ century, A. baumannii emerged as one of the most challenging pathogens due to its high adaptation to hospital environments (3). This microorganism has been cited among the six top priority dangerous drug-resistant bacteria by Infectious Diseases Society of America (4), as its prevalence is becoming an alarming all over the world(5). The epidemiological studies concerning the MDR A. baumannii strains show the dissemination of different healthcare-associated MDR $A$. baumannii clones worldwide(6).

Enzyme alteration, the permeability of the outer membrane, the mutation in target genes, increased expression of efflux pumps and mobile genetic elements are the key antibiotic resistance mechanisms in A. baumannii microorganism (7). Among them, integrons have been known as the chief source of resistance genes in Gram-negative bacteria including $A$. baumannii (8). Integrons are DNA elements capable of capturing genes by a site-specific recombination mechanism that often carries gene cassettes containing antibiotic resistance genes (9). Until now, six classes of integrons (according to intl gene) have been identified, in which classes 1,2 and 3 showed a major role in transferring antibiotic resistance genes (10). Class 1 integrons are usually expressed in $A$. baumannii and play a significant role in antibiotic resistance and typically encode genes for aminoglycoside resistance, $\beta$-lactamases, Metallo- $\beta$-lactamases, and oxacillinases (11). Class 2 integrons are carried out inside the transposon $\operatorname{Tn} 7$ with gene cassettes and attributed in conferring resistance to chloramphenicol, trimethoprim, aminoglycosides, and streptomycin (12).

To our knowledge, determination of integrons prevalence in clinical isolates of $A$. baumannii provides appropriate information concerning the pattern of antibiotic resistance in each region. In the current study, due to low importance of class 2 and 3 integrons in antibacterial resistance and because of the lack of reporting these integrons in studies included in the review, this study aimed to investigate the prevalence of integrons and antibiotic resistance pattern in $A$. baumannii isolated from clinical samples of Iranian patients through systematic review and meta-analysis.

\section{METHODS}

Information source and search strategy: The Medical Subject Headings (MeSH) and tht keywords of "Integrons", "Int1", "prevalence", "antibiotic resistance", "A. baumannii", "Integron prevalence", and "Iran, with the help of Boolean operators ("AND" or "OR") were used alone or in combinations to conduct a broad search. The searching process was done in the Web of Science, PubMed, Cochrane Library, Scopus and Google Scholar databases. The search was restricted to the papers published in either English or Persian from 1 January 2000 to 31 December 2018. The studies reporting the prevalence, or distribution of Int1 in A. baumannii isolated from clinical samples of Iranian patients were included in the present review. Correspondingly, the Persian equivalents of the keywords were used in a comparable strategy to recover Persian articles in national databases such as Magiran (www.Magiran.com), Irandoc (www.irandoc.ac.ir), Iranmedex (www.iranmedex.com), and Scientific Information Database (www.sid.ir).

Inclusion and exclusion criteria: All relevant English and Persian cross-sectional and cohort publications reporting the prevalence of Int1 in $A$. baumannii isolated from clinical samples of Iranian patients and cited by databases were retrieved and included in the current review. All review forms including (narrative, meta-analysis or systematic), prospective studies, congress, case reports, meeting, abstracts, studies in languages other than English or Persian, editorials, and letters to the editors were excluded. Furthermore, studies without full text and duplicate publications were considered as excluded criteria, too. Two independent reviewers completed all these search processes to decrease the risk of error and bias.

DOI: http://dx.doi.org/10.4314/ejhs.v29i5.2 
Screening: All recognized articles in both languages from the mentioned databases were entered into our created file to eliminate duplicates. After that, the documents were screened by two reviewers (HV and FJJ) independently in a few levels. These levels screen included title screening, abstract screening and lastly full-text screening. At each level, the investigators individually assessed the studies, and then shared their agreements in the common file. Disagreements were resolved by discussion before including the studies for the next screening level. Dissimilarities between the assessors were argued and resolved through agreement. Where no agreement was reached, a third reviewer was involved in decision-making. Finally, records were assessed for met eligibility and final selection.

Quality assessment: For quality assessment of studies methodology was used of the criteria mentioned in Critical Appraisal Skills Programmed checklists (www.casp-UK). As for each study independently, a series of main questions were designed. If the relevant information was clearly reported, a question was scored as 'yes'. If no, or in case of any doubt, the question was marked as 'no' or 'can't tell'. Overall, studies were classified as 'strong', 'moderate', or 'weak', according to the number of questions scored as 'yes'(13). At last, weak studies were removed from the current review.

Assessing the risk of bias: For quality assessment and prevention of the risk of bias, several items including design, method, context, findings, and analyses of the study, also, interpretations of the findings were evaluated. The studies with a key flaw in the considered items were excluded. Also, it should be noted that any conflicts on the quality evaluation or the risk of bias in recording, reporting, collection, contributors, extraction of data, confusing factors, and statistical analyses were resolved by debates between the two assessors or by referring to a third reviewer (14).

Data extraction: The data were extracted from each study by a data extraction form designed for the reviewers. The extracted data included (a) the first author's name, (b) publication year, (c) study setting, (d) sample size, (e) MDR prevalence, (f) and Int 1 prevalence.

Data analysis: The data were analyzed using Comprehensive Meta-Analysis software (Version 3.3.070). The prevalence was presented by a $95 \%$ Confidence Interval (CI). Regarding the heterogeneity of studies, the random-effects model was used for meta-analysis. The Cochrane Q and $I^{2}$ tests were used to evaluate the statistical heterogeneity between the studies. To estimate possible publication bias, quantitative Egger weighted regression test and funnel plot were used. Also, $P$-value $<0.05$ was considered as the statistical significance threshold. Additionally, the asymmetrical distribution of the studies was evaluated by Egger's linear regression test.

\section{RESULTS}

Study inclusion: The study selection route is abstracted in Figure 1. In brief, 586 relevant records were firstly identified in the initial search in scientific databases. Two hundred-eleven duplicates were removed. Regarding assessing the abstracts of the 375 studies, 286 literatures were excluded. Then, full texts of 89 studies were assessed for eligibility. About 74 articles were excluded due to lack of sufficient data and not reporting Int1 prevalence. Finally, 15 studies were included in the present systematic review and meta-analysis. 

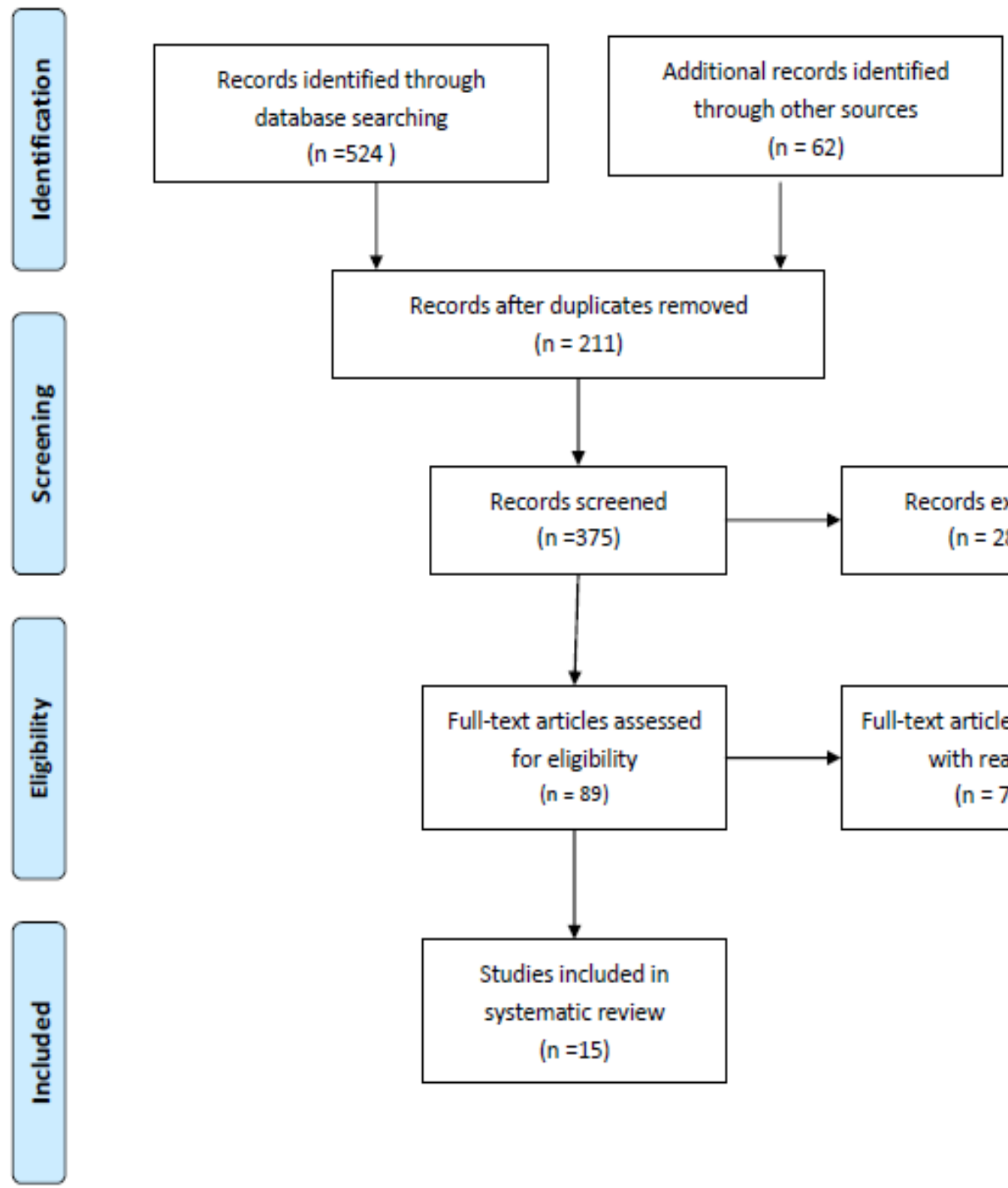

Records after duplicates removed

$(n=211)$
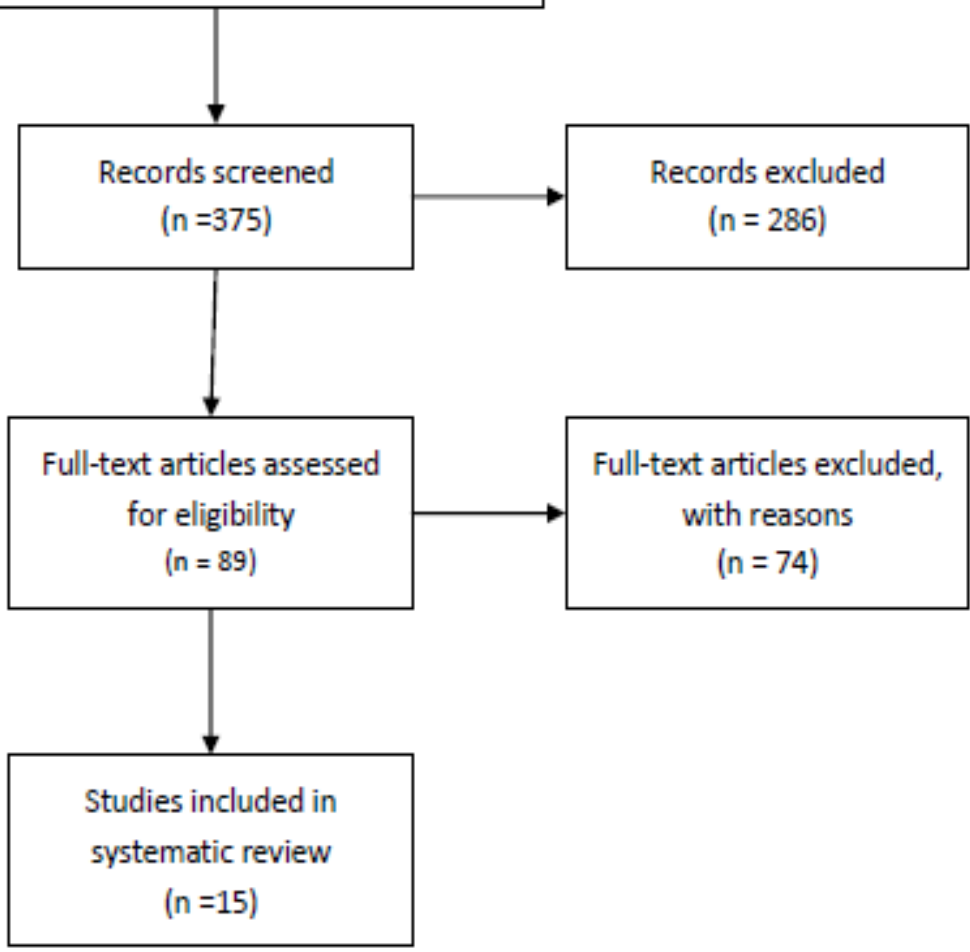

Figure1: Flow chart diagram of studies included in the present review

Characteristics of the included studies: As presented in Table 1, the selected articles covered various areas of Iran (i.e. Center, Northwest, West, and South). The prevalence of Int1 in $A$. baumannii isolated from clinical samples of Iranian patients varied from $12 \%$ to $98.4 \%$ (Figure $2)$. The highest prevalence of Int1 was reported in a study conducted by Japoni-Nejad et al.(15) with a frequency of $98.4 \%$. The frequency of MDR $A$. baumannii isolates varied from $29 \%$ to $100 \%$. As shown in Figure 3, the publication bias was checked using Funnel plot. Regarding possible asymmetrical data distribution in included studies, the findings of the Egger's linear regression test did not show any publication bias $(P=0.6)$.

DOI: http://dx.doi.org/10.4314/ejhs.v29i5.2 
Table 1: Characteristics of included studies in clinical specimens.

\begin{tabular}{lllcll}
\hline Study & $\begin{array}{l}\text { Publication } \\
\text { (Year) }\end{array}$ & Location & Sample Size & $\begin{array}{l}\text { MDR } \\
\text { prevalence }\end{array}$ & Int1 prevalence \\
\hline Asadollahi (32) & 2011 & Tehran & 40 & $40(100 \%)$ & $27(67.5 \%)$ \\
Taherikalani et al(33) & 2011 & Tehran & 100 & - & $58(58 \%)$ \\
Asadollahi et al(34) & 2012 & Tehran & 23 & - & $13(56.5 \%)$ \\
Farajnia et al(35) & 2013 & Tabriz & 100 & $80(80 \%)$ & $74(74 \%)$ \\
Mirnejad et al(36) & 2013 & Tehran & 50 & $41(82 \%)$ & $15(21 \%)$ \\
Japoni-Nejad et al(15) & 2013 & Arak & 63 & - & $62(98.4 \%)$ \\
Kamalbeik et al(37) & 2014 & Tehran & 42 & - & $5(12 \%)$ \\
Azizi et al(38) & 2016 & Kerman & 65 & $65(100 \%)$ & $46(70.8 \%)$ \\
Goudarzi (39) & 2016 & Tehran & 120 & $120(100 \%)$ & $89(74.2 \%)$ \\
Goudarzi et al(40) & 2017 & Tehran & 105 & $105(100 \%)$ & $70(66.7 \%)$ \\
Eghbalimoghadam(41) & 2017 & Kermanshah & 100 & $29(29 \%)$ & $42(42 \%)$ \\
Mirshekar et al(42) & 2018 & Tehran & 72 & - & $42(58.3 \%)$ \\
Halaji et al(43) & 2018 & Isfahan & 147 & - & $94(63.9 \%)$ \\
Eftekhar et al(44) & 2018 & Tehran & 50 & - & $11(22 \%)$ \\
Shaheli et al(45) & 2018 & Shiraz & 83 & $77(92.9 \%)$ & $27(32.5 \%)$ \\
\hline
\end{tabular}

Table 2: Analysis of prevalence of class 1 integron in P. aeruginosa recovered from Iranian burn patients

\begin{tabular}{lccccccccc}
\hline Subgroups & $\begin{array}{c}\text { Number } \\
\text { of } \\
\text { studies }\end{array}$ & \multicolumn{3}{c}{ Heterogeneity test } & \multicolumn{2}{c}{ Egger's test } & \multicolumn{2}{c}{ Random model } \\
\cline { 3 - 9 } & $\begin{array}{l}\text { Prevalence } \\
(95 \% \mathrm{CI}) \\
(\%)\end{array}$ & & $\mathrm{P}$ & $\mathrm{Q}$ & $\mathrm{P}$ & $\mathrm{I}^{2}$ & $\mathrm{~T}$ & $\mathrm{P}$ \\
& 15 & $\begin{array}{l}55.2(44.8- \\
65.1)\end{array}$ & 0.97 & 0.00 & 137 & 0.60 & 92 & 0.53 & 0.37 \\
\hline $\begin{array}{l}\text { Overall } \\
\text { effect(Int1) }\end{array}$ & 8 & $\begin{array}{l}68.1(61.9- \\
\text { MDR }\end{array}$ & 5.4 & 0.00 & 121.3 & 0.04 & 94.2 & 6 & 0.04 \\
\hline
\end{tabular}

Overall effects: Regarding the heterogeneity test, the values were attained as $\mathrm{Q} 2=137, \mathrm{I}^{2}=92, \mathrm{t}=0.5$ $(P=0.6)$ for the selected studies. Therefore, according to the findings of heterogeneity test, the random effects model was used to combine the data on prevalence of class 1 integron in $A$. baumannii recovered from clinical samples. The combined prevalence of class 1 integrons in $A$. baumannii recovered from clinical samples was $55.2 \% \quad(95 \%$ CI: 44.8-65.1) (Figure 2). The clinical samples were mostly collected from ICU and other wards. The pooled prevalence of MDR
A. baumannii isolates was $68.1 \%$ (95\% CI: 61.9 73.7). As presented in Table 3, subgroups analysis of antibiotic resistance pattern of $A$. baumannii revealed that the highest combined resistance belonged to Aztreonam, followed by Ciprofloxacin, and Ceftazidime with a resistance rate of $97.6 \%, 92.8 \%$, and $91.6 \%$, respectively. As well as, the least resistance was against Tobramycin with a resistance rate of $38.7 \%$. The detailed information about antibiotic susceptibility is listed in Table 3. 


\section{Int1}

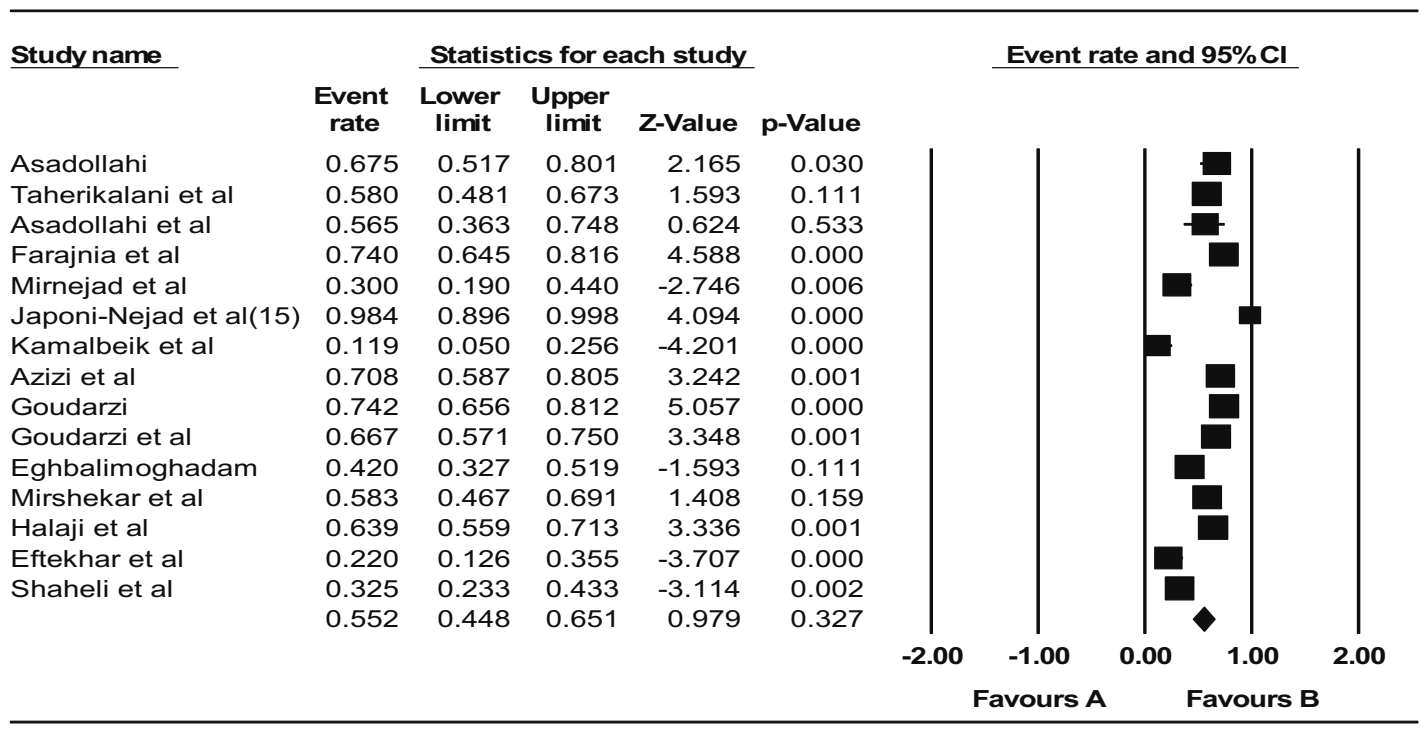

Figure 2: Forest plot of the meta-analysis of prevalence of class 1 integron in A. baumannii isolated from clinical samples

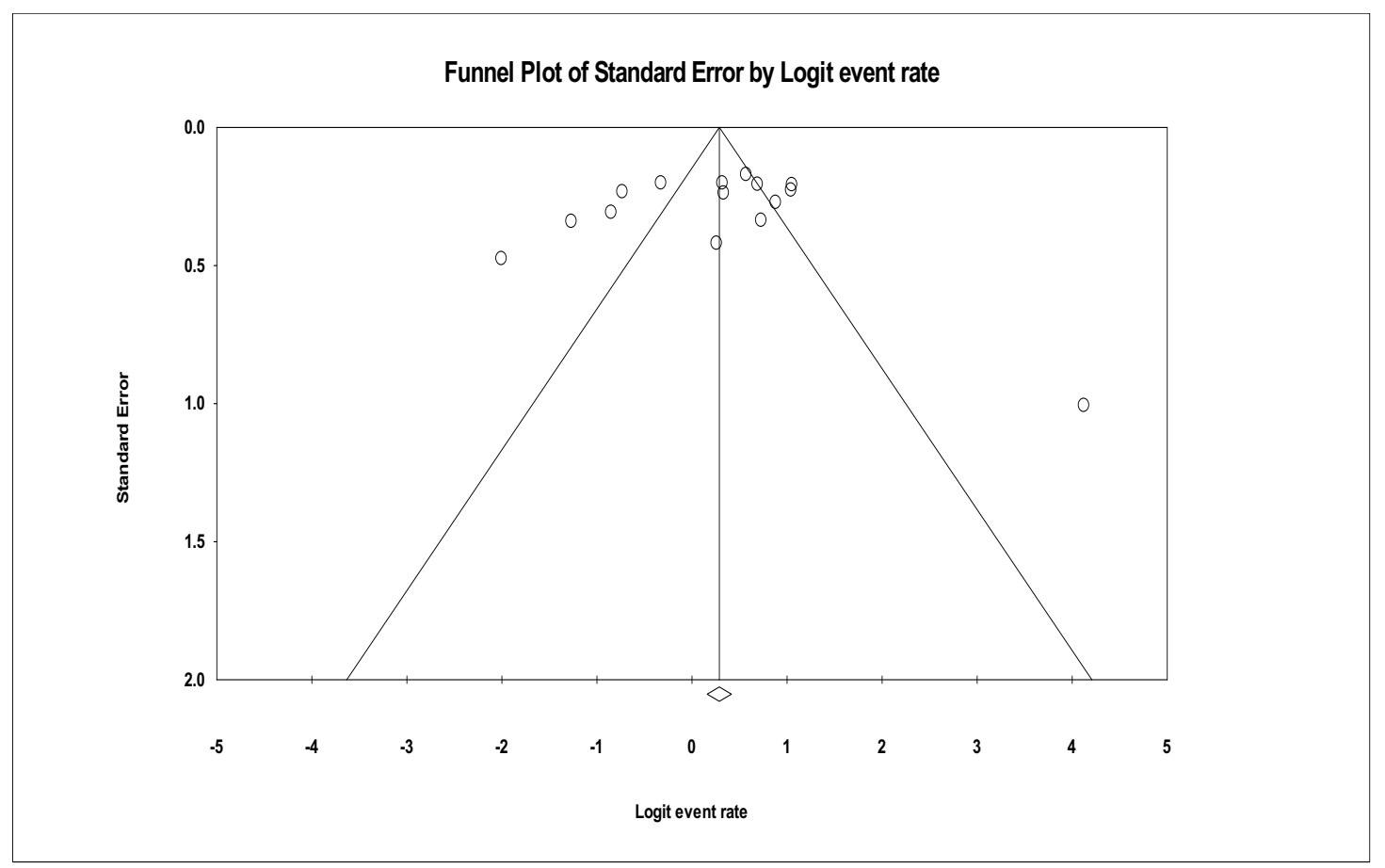

Figure 3: Funnel plot of meta-analysis on the prevalence of class 1 integron in A. baumannii recovered from clinical samples

DOI: http://dx.doi.org/10.4314/ejhs.v29i5.2 
Table 3: Subgroups analysis for antibiotic resistance in P. aeruginosa isolated from Iranian burn patients

\begin{tabular}{|c|c|c|c|c|c|c|c|c|c|}
\hline \multirow[t]{2}{*}{ Subgroups } & \multirow{2}{*}{$\begin{array}{l}\text { Number } \\
\text { of } \\
\text { studies }\end{array}$} & \multicolumn{2}{|c|}{ Heterogeneity test } & \multicolumn{3}{|c|}{ Egger's test } & \multicolumn{3}{|c|}{ Random model } \\
\hline & & $\begin{array}{l}\text { Prevalence } \\
(95 \% \mathrm{CI}) \\
(\%)\end{array}$ & Z & $\mathrm{P}$ & Q & $\mathrm{P}$ & $\mathrm{I}^{2}$ & $\mathrm{~T}$ & $\mathrm{P}$ \\
\hline Imipenem & 14 & $\begin{array}{l}75.9(63.5- \\
85.1)\end{array}$ & 3.7 & 0.00 & 171.5 & 0.00 & 92.4 & 2.1 & 0.05 \\
\hline Ciprofloxacin & 14 & $\begin{array}{l}92.8(85.2- \\
96.6)\end{array}$ & 6.2 & 0.00 & 161.8 & 0.00 & 91.9 & 2.8 & 0.01 \\
\hline Gentamicin & 15 & $\begin{array}{l}72.5(61.3- \\
81.5)\end{array}$ & 3.7 & 0.00 & 191.7 & 0.00 & 92.6 & 1.9 & 0.07 \\
\hline Amikacin & 11 & $\begin{array}{l}74.2(58.1- \\
85.6)\end{array}$ & 2.8 & 0.005 & 197.3 & 0.00 & 94.9 & 1.1 & 0.26 \\
\hline Ceftriaxone & 7 & $\begin{array}{l}90.6(73.1- \\
97.2)\end{array}$ & 3.5 & 0.00 & 119.5 & 0.00 & 94.9 & 2.5 & 0.05 \\
\hline Ceftazidime & 11 & $\begin{array}{l}91.6(80.3- \\
96.7)\end{array}$ & 4.7 & 0.00 & 187.9 & 0.00 & 94.6 & 5.7 & 0.00 \\
\hline Cefepime & 10 & $\begin{array}{l}56.3(51.3- \\
61.7)\end{array}$ & 2.4 & 0.00 & 105.2 & 0.00 & 91.4 & 8.6 & 0.00 \\
\hline $\begin{array}{l}\text { Piperacillin/ } \\
\text { tazobactam }\end{array}$ & 11 & $\begin{array}{l}59.7(55.6- \\
63.7)\end{array}$ & 4.5 & 0.00 & 131.5 & 0.00 & 92.3 & 3.9 & 0.00 \\
\hline Aztreonam & 2 & $\begin{array}{l}\text { 97.6(95.1- } \\
98.9)\end{array}$ & 9.7 & 0.00 & 0.5 & 0.000 & 0.00 & 0.00 & 0.00 \\
\hline Tobramycin & 2 & $\begin{array}{l}38.7(19.5- \\
62.2)\end{array}$ & 0.94 & 0.34 & 16.4 & 0.00 & 93.9 & 0.00 & 0.00 \\
\hline Cefotaxime & 11 & $\begin{array}{l}\text { 65.6(60.4- } \\
70.4)\end{array}$ & 5.6 & 0.00 & 106.3 & 0.00 & 91.5 & 3.8 & 0.00 \\
\hline
\end{tabular}

\section{DISCUSSION}

A. baumannii has an important role in nosocomial infection owing to its resistance to different classes of antibiotics (16). This resistance is caused by different mechanisms. Among them, Integrons plays an important role, and spreading of antibiotic resistance genes via them is problematic in treatment of infections caused by this microorganism $(4,17)$. The findings of the current study showed the combined prevalence of $55.2 \%$ of class 1 integrons in $A$. baumannii recovered from clinical specimens. The pooled prevalence of MDR A. baumannii isolates was $68.1 \%$. The prevalence of class 1 integrons are increased all over the world. In aagreement with our findings, other reports from Italy (18), and China (19) showed the prevalence $44 \%$ and $71 \%$, respectively. However, Javier Ariza, et al. reported a lower prevalence (25\%) from Spain (20).

This high prevalence of integron class 1 in $A$. baumannii isolates is attributed to several reasons such as inappropriate use of antibiotics for treatment of $A$. baumannii infections, and consequently, the high expression of gene cassettes containing integrons class 1 and, secondly, the ability of integrons to acquire new gene cassettes lead to disseminating antibiotic resistance among clinical isolates. Finally, failure in establishment of a national surveillance program as part of the Global Strategy for Containment of Antimicrobial Resistance in medical centers leads to survival of MDR $A$. baumannii isolates carrying integron and spreading of resistance integrons among other $A$. baumannii isolates (21). Class 1 integrons are prevalent as compared to the other classes of

DOI: http://dx.doi.org/10.4314/ejhs.v29i5.15 
integrons possibly due to the location of class 1 integrons on genetic elements such as conjugative plasmids and transposons (22). As stated in the present review, the pooled prevalence of MDR $A$. baumannii isolates was $68.1 \%$.

This subject leads to MDR A. baumannii isolates carrying integron and spreading of resistance integrons among other A. baumannii isolates. Inconsistent with the present review, another review from Iran showed that the frequency of MDR A. baumannii isolates increased from $50 \%$ in $2001-2007$ to $74 \%$ in 2010-2015, with a mean prevalence of 71\% (23). Prolonged hospital stay especially in Intensive Care Unit (ICU), receiving of ventilator, recent surgery, medical interventions, colonization pressure, and use of overdose of antibiotics such as Carbapenems, Colistin, invasive procedures, underlying diseases are the main risk factors for colonization or infection with MDR A. baumannii isolates (24). To our knowledge, Imipenem acts as a choice drug in the treatment of infections caused by $A$.baumannii (5). As shown in the results section, the resistance rate against Imipenem was reported as $75.9 \%$. The resistance against Imipenem is increased worldwide as some studies from other countries including Pakistan (100\%), Turkey (98\%), United Arab Emirates $(76 \%)$ and Saudi Arabia (63\%) reported the same pattern (25-28). In confirmation of our results, a study from Taiwan revealed that MDR-AB isolates carrying integrons were significantly more resistant to almost all antibiotics used than nonintegron-carrying isolates (19). In geographical districts including China and Spain, Integroncarrying MDR-AB isolates have been reported, too $(29,30)$. Their results showed that MDR-AB strains carrying different gene cassettes including different integron cassette types 2, 3, 4 and 6 have been found to have similar multidrug resistance patterns, indicating the correlation of integrons and multidrug resistance. Then, we can conclude that the high MDR isolates in the current review can possibly be attributed to the existence of class 1 integrons. Clinical isolates of $A$. baumannii all over the world share different resistance mechanisms with other bacterial genera (30). Therefore, the high prevalence of the Integrons in clinical isolates of $A$. baumannii from Iran can be very worrying, and A. baumannii isolates will not respond to existing antibiotics any more and cause transmission of resistance through the Integrons. Establishing a national surveillance program as part of the Global Strategy for Containment of Antimicrobial Resistance in medical centers is critical (31). When antibiotics become ineffective due to bacterial resistance, implement, and evaluate new specific containment interventions is crucial to collect specific information regarding MDR isolates of $A$. baumannii.

The present study reported alarming high prevalence of class 1 Integrons and MDR isolates of $A$. baumannii are recovered from clinical samples that should be considered.

\section{ACKNOWLEDGMENTS}

We, the authors, would like to thank our colleagues for their help in this work.

\section{REFERENCES}

1. Bahador A, Raoofian R, Farshadzadeh Z, Beitollahi L, Khaledi A, Rahimi S, et al. The prevalence of ISAba1 and ISAba4 in Acinetobacter baumannii species of different international clone lineages among patients with burning in Tehran, Iran. Jundishapur J Microbiol. 2015;8(7):1-9.

2. Saghi H, Bahador A, Khaledi A, Ataee Kr, Amiri Df, Esmaeili D. Antibacterial effects of Origanum vulgare essence against multidrug-resistant Acinetobacter baumannii isolated from selected hospitals of Tehran, Iran. J Pure Appl Med Sci. 2015.16-25.

3. Bahador A, Bazargani A, Taheri M, Hashemizadeh $\mathrm{Z}$, Khaledi A, Rostami H, et al. Clonal lineages and virulence factors among Acinetobacter baumannii isolated from Southwest of Iran. J Pure Appl Micribiol. 2013;7:1559-66.

4. Khaledi A, Fatemeh D, Hosseini SMJ, Meskini M, Esmaeili D. Antimicrobial Resistance Pattern of Acinetobacter baumannii Strains Isolated from Intensive Care Unit Patients. $J$ Pure Appl Micribiol. 2018. 32-44.

5. Khaledi A, Esmaeili D, Jamehdar SA, Esmaeili SA, Neshani A, Bahador A. Expression of MFS efflux pumps among multidrug resistant Acinetobacter baumannii clinical isolates. Der Pharm Lett. 2016;8:262.

6. Khaledi A, Elahifar O, Vazini H, Alikhani MY, Bahrami A, Esmaeili D, et al. Increasing Trend of Imipenem-Resistance Among Acinetobacter

DOI: http://dx.doi.org/10.4314/ejhs.v29i5.2 
baumannii Isolated From Hospital Acquired Pneumonia in Northeast of Iran. Avicenna J Clin Microbiol Infect. 2017;4(3):231-39.

7. Zarifi E, Eslami G, Khaledi A, Vakili M, Vazini H, Zandi $\mathrm{H}$. Prevalence of ESBLs in Acinetobacter baumannii isolated from intensive care unit (ICU) of Ghaem hospital, Mashhad, Iran. J Pure Appl Microbiol. 2017;11(2):811-9.

8. Nemec A, Dolzani L, Brisse S, van den Broek P, Dijkshoorn L. Diversity of aminoglycosideresistance genes and their association with class 1 integrons among strains of pan-European Acinetobacter baumannii clones. J Medi Microbiol. 2004;53(12):1233-40.

9. Navia MM, Ruiz J, Vila J. Characterization of an integron carrying a new class D $\beta$-lactamase (OXA-37) in Acinetobacter baumannii. Microb Drug Resist. 2002;8(4):261-5.

10. Huang C, Long Q, Qian K, Fu T, Zhang Z, Liao P, et al. Resistance and integron characterization of Acinetobacter baumannii in a teaching hospital in Chongqing, China. New Microbes New Infect. 2015;8:103-8.

11. Zhao W-H, Hu Z-Q. IMP-type metallo- $\beta$ lactamases in Gram-negative bacilli: distribution, phylogeny, and association with integrons. Crit Rev Microbiol. 2011;37(3):214-26.

12. Bonnin RA, Nordmann P, Potron A, Lecuyer H, Zahar J-R, Poirel L. Carbapenem-hydrolyzing GES-type extended-spectrum $\beta$-lactamase in Acinetobacter baumannii. Antimicrob Agents Chemother. 2011;55(1):349-54.

13. CASP. 10 questions to help you make sense of qualitative research. 2006. 342-47.

14. Auguste P, Tsertsvadze A, Pink J, McCarthy N, Sutcliffe P, Clarke A. Comparing interferongamma release assays with tuberculin skin test for identifying latent tuberculosis infection that progresses to active tuberculosis: systematic review and meta-analysis. BMC Infect Dis. 2017;17(1):200.

15. Japoni-Nejad A, Farshad S, van Belkum A, Ghaznavi-Rad E. Novel cassette array in a class 1 integron in clinical isolates of Acinetobacter baumannii from central Iran. Int J Med Microbiol. 2013;303(8):645-50.

16. Perez F, Hujer AM, Hujer KM, Decker BK, Rather PN, Bonomo RA. Global challenge of multidrugresistant Acinetobacter baumannii. Antimicrob Agents Chemother. 2007;51(10):3471-84.

17. Moubareck C, Brémont S, Conroy M-C, Courvalin $\mathrm{P}$, Lambert T. GES-11, a novel integron-associated GES variant in Acinetobacter baumannii.
Antimicrob Agents Chemother. 2009;53(8):357981.

18. Gombac F, Riccio ML, Rossolini GM, Lagatolla C, Tonin E, Monti-Bragadin C, et al. Molecular characterization of integrons in epidemiologically unrelated clinical isolates of Acinetobacter baumannii from Italian hospitals reveals a limited diversity of gene cassette arrays. Antimicrob Agents Chemother. 2002;46(11):3665-8.

19. Huang L-Y, Chen T-L, Lu P-L, Tsai C-A, Cho WL, Chang F-Y, et al. Dissemination of multidrugresistant, class 1 integron-carrying Acinetobacter baumannii isolates in Taiwan. Clin Microbiol Infect. 2008;14(11):1010-9.

20. Ribera A, Vila J, Fernández-Cuenca F, MartínezMartínez L, Pascual A, Beceiro A, et al. Type 1 integrons in epidemiologically unrelated Acinetobacter baumannii isolates collected at Spanish hospitals. Antimicrob Agents Chemother. 2004;48(1):364-5.

21. Pormohammad A, Pouriran R, Azimi H, Goudarzi M. Prevalence of integron classes in Gramnegative clinical isolated bacteria in Iran: a systematic review and meta-analysis. Iran J Basic Med Sci. 2019;22(2):118.

22. Mazel D. Integrons: agents of bacterial evolution. Nat Rev Microbiol. 2006;4(8):608.

23. Pourhajibagher M, Hashemi FB, Pourakbari B, Aziemzadeh M, Bahador A. Antimicrobial resistance of Acinetobacter baumannii to imipenem in Iran: a systematic review and meta-analysis. Open Microbiol J. 2016;10:32.

24. Dijkshoorn L, Nemec A, Seifert H. An increasing threat in hospitals: multidrug-resistant Acinetobacter baumannii. Nat Rev Microbiol. 2007;5(12):939.

25. Begum S, Hasan F, Hussain S, Shah AA. Prevalence of multi drug resistant Acinetobacter baumannii in the clinical samples from Tertiary Care Hospital in Islamabad, Pakistan. Pakistan J Med Sci. 2013;29(5):1253.

26. Güven T, Yilmaz G, Güner Hr, Kalem Ak, Eser F, Taşyaran Ma. Increasing resistance of nosocomial Acinetobacter baumannii: are we going to be defeated? Turkish J Med Sci. 2014;44(1):73-8.

27. Sonnevend Á, Ghazawi A, Al Munthari N, Pitout M, Hamadeh MB, Hashmey R, et al. Characteristics of epidemic and sporadic strains of Acinetobacter baumannii isolated in Abu Dhabi hospitals. J Med Microbiol. 2013;62(4):582-90.

28. Al-Agamy MH, Shibl AM, Ali MS, Khubnani H, Radwan HH, Livermore DM. Distribution of $\beta$ lactamases in carbapenem-non-susceptible

DOI: http://dx.doi.org/10.4314/ejhs.v29i5.15 
Acinetobacter baumannii in Riyadh, Saudi Arabia. J Glob Antimicrob Resist. 2014;2(1):17-21.

29. Gu B, Tong M, Zhao W, Liu G, Ning M, Pan S, et al. Prevalence and characterization of class I integrons among Pseudomonas aeruginosa and Acinetobacter baumannii isolates from patients in Nanjing, China. J Clin Microbiol. 2007;45(1):241.

30. Gallego L, Towner KJ. Carriage of class 1 integrons and antibiotic resistance in clinical isolates of Acinetobacter baumannii from northern Spain. J Med Microbiol. 2001;50(1):71-7.

31. Gonzalez-Villoria AM, Valverde-Garduno V. Antibiotic-resistant Acinetobacter baumannii increasing success remains a challenge as a nosocomial pathogen. J pathog. 2016;2016.

32. Asadollahi K, Taherikalani M, Maleki A, Alizadeh E, Valadbaigi H, Soroush S, et al. Diversity of aminoglycoside modifying enzyme genes among multidrug resistant Acinetobacter baumannii genotypes isolated from nosocomial infections in Tehran hospitals and their association with class 1 integrons. Acta Microbiol Immunol Hung. 2011;58(4):359-70.

33. Taherikalani M, Maleki A, Sadeghifard N, Mohammadzadeh D, Soroush S, Asadollahi P, et al. Dissemination of class 1,2 and 3 integrons among different multidrug resistant isolates of Acinetobacter baumannii in Tehran hospitals. Iran Pol J Microbiol. 2011;60(2):169-74.

34. Asadollahi P, Akbari M, Soroush S, Taherikalani M, Asadollahi K, Sayehmiri K, et al. Antimicrobial resistance patterns and their encoding genes among Acinetobacter baumannii strains isolated from burned patients. Burns. 2012;38(8):1198-203.

35. Farajnia S, Azhari F, Alikhani MY, Hosseini MK, Peymani A, Sohrabi N. Prevalence of PER and VEB type extended spectrum betalactamases among multidrug resistant Acinetobacter baumannii isolates in North-West of Iran. Iran $J$ Basic Med Sci. 2013;16(6):751.

36. Mirnejad R, Mostofi S, Masjedian F. Antibiotic resistance and carriage class 1 and 2 integrons in clinical isolates of Acinetobacter baumannii from Tehran, Iran. Asian Pac $J$ Trop Biomed. 2013;3(2):140-5.

37. Kamalbeik S, Talaie H, Mahdavinejad A, Karimi A, Salimi A. Multidrug-resistant Acinetobacter baumannii infection in intensive care unit patients in a hospital with building construction: is there an association? Korean $J$ Anesthesiol. 2014;66(4):295.

38. Azizi O, Shakibaie MR, Badmasti F, Modarresi F, Ramazanzadeh R, Mansouri S, et al. Class 1 integrons in non-clonal multidrug-resistant Acinetobacter baumannii from Iran, description of the new blaIMP-55 allele in In1243. $J$ Med Microbiol. 2016;65(9):928-36.

39. Goudarzi H, Azad M, Seyedjavadi SS, Azimi H, Chirani AS, Omrani VF, et al. Characterization of integrons and associated gene cassettes in Acinetobacter baumannii strains isolated from intensive care unit in Tehran, Iran. $J$ Acute Dis. 2016;5(5):386-92.

40. Goudarzi M, Azimi H. Dissemination of classes 1, 2 , and 3 integrons in acinetobacter baumannii strains recovered from intensive care units using polymerase chain reaction-restriction fragment length polymorphism. Jundishapur $J$ Microbiol. 2017;10(5): 45-53.

41. Eghbalimoghadam M, Farahani A, Akbar FN, Mohajeri P. Frequency of Class 1 integron and genetic diversity of Acinetobacter baumannii isolated from medical centers in Kermanshah. $J$ Nat Sci Biol Med. 2017;8(2):193.

42. Mirshekar M, Shahcheraghi F, Azizi O, Solgi H, Badmasti F. Diversity of class 1 integrons, and disruption of carO and dacD by insertion sequences among Acinetobacter baumannii isolates in Tehran, Iran. Microb Drug Resist. 2018;24(4):359-66.

43. Halaji M, Rezaei A, Zalipoor M, Faghri J. Investigation of class I, II, and III integrons among Acinetobacter Baumannii isolates from hospitalized patients in Isfahan, Iran. Oman Med J. 2018;33(1):37.

44. Eftekhar F, Altayar F, Khidaei H. Plasmidmediated class 1 and 2 integron carriage in drugresistant nosocomial isolates of Acinetobacter baumannii. Arch Clin Infect Dis. 2018;13(1):123-9.

45. Shaheli M, Salehi BM, Bahador N. The influence of integrons on multidrug resistant Acinetobacter spp. isolated from environment and clinical samples. Trop Biomed. 2018;35(2):354-64. 